traditional risk factors (smoke, hypertension, dyslipidemia) and treatment with aspirin and hydroxychloroquine.

Conclusion Our results confirmed that Italian lupus patients suffer a high incidence of CV disease compared with general population. However, this incidence was lower than that detected in North European and American lupus cohorts

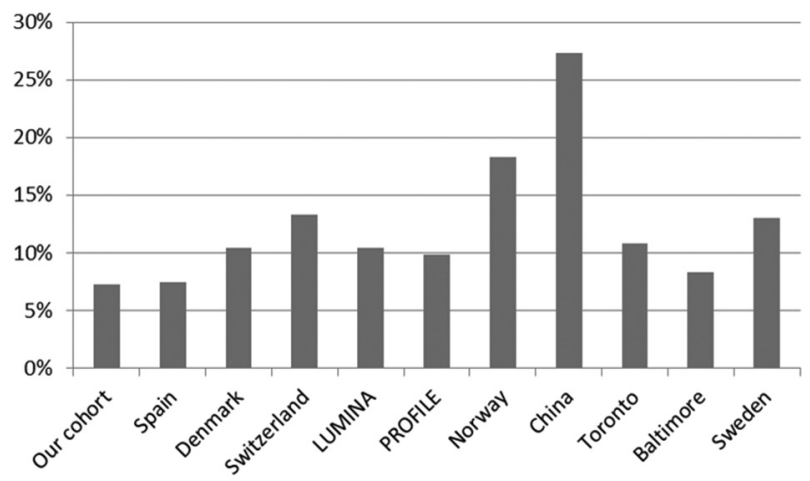

Abstract PS3:48 Figure 1

\section{PS3:49 EVALUATION OF CAPILLAROSCOPIC PATTERN IN SLE PATIENTS WITH AND WITHOUT RAYNAUD SYMPTOM}

G Nagy, L Czirják, G Kumánovics. University of Pécs, Department of Rheumatology and Immunology, Pécs, Hungary

\subsection{6/lupus-2018-abstract.97}

Background Capillaroscopy is a noninvasive method for evaluating nailfold abnormalities and differentiating between primary and secondary Raynaud syndrome (RP). It is widely investigated in systemic sclerosis (SSc) but not in systemic lupus erythematosus (SLE). SSc pattern is described with decreased capillary density, haemorrhage, neoangiogenesis and avascularity.

Objective Evaluate capillaroscopic pattern and clinical features in SLE patients; examine the influence of RP on capillaroscopic pattern and capillary density.

Methods 318 systemic autoimmune patients and 25 healthy controls were collected, 73 fulfilled SLE classification criteria. All patients underwent detailed nailfold capillaroscopic investigation. Density, intercapillary distance was recorded as well as the progression and diagnostic parameters described by Cutulo in semiquantitative manner. Presence of RP was investigated by a detailed questionnaire. Patients with and without RP were compared. 89 patients fulfilled SSc classification criteria, the median capillary density was $6.66(5.2 ; 7.94)$ in this group, the median microangiopathia evaluation score (MES) was $1.97(1.19 ; 3.13)$ in SSc subgroup.

Results 23 patients had pure 'idiopathic' SLE, 36 fulfilled SLE plus another classification criteria, 11 SLE plus two other, 2 SLE plus three other and 1 SLE plus four other. Median capillary density was $8.23(7.4 ; 8.94)$, the median MES was $1.00(0.56 ; 1.47)$; the median giant capillary number was $0.00(0.00 ; 0.75)$ in the entire SLE group. $6.9 \%$ of all SLE patients had SSc early pattern, $1.4 \%$ SSc active pattern, $20.6 \%$ had SSc late pattern and $71.2 \%$ had no SSc pattern. Among patients having SSc pattern all except two had RP. Comparison of capillaroscopy of SLE patients with and without RP showed that patients in the former group had significantly lower capillary density (7.97 [7.19; 8.72] vs. 8.92 $(8.19 ; 9.34), \mathrm{p}<0.05)$. Dilatation point and giant capillary point was significantly higher in the RP-SLE subgroup $(0.36$ $[0.13 ; 0.69]$ vs $0.13[0.06 ; 0.28] \mathrm{p}<0.05,0.06[0.00 ; 0.28$ vs. $0.00[0.00 ; 0.00] \mathrm{p}<0.001)$.

Conclusion SSc capillary pattern is present in SLE as well, most of these particular patients had Raynaud's phenomenon. Patients having both SLE and RP have lower capillary density and worse capillary structure. SLE patients capillary density is higher than the density found in SSc controls.

\section{PS3:50 INCIDENCE, DISEASE SEVERITY AND OUTCOME OF LUPUS NEPHRITIS. RESULTS FROM AN INCEPTION COHORT OF HISPANIC SLE PATIENTS}

JV Medina-Gomez, MC Ocampo-Torres, P Lara-Reyes, Z Garcia-Sanabria, J Romero-Diaz. Instituto Nacional de Cs Medicas y Nutricion S.Z. Department of Immunology and Rheumatology, Mexico City, Mexico

\subsection{6/lupus-2018-abstract.98}

Background Lupus nephritis among Hispanic SLE patients have been identified with poor outcomes when it is compared to other populations; so, we aimed to identify lupus nephritis characteristics and its outcomes in an inception cohort of Hispanic SLE patients.

Patients and methods two-hundred twenty-three patients with SLE of recent-onset were studied. At baseline, standardised medical history and laboratory tests were done; followup visits occurred quarterly, and information about renal disorder, disease activity, damage accrual and comorbidities was updated annually. Main outcome was the development of renal disorder since SLE diagnosis, incidence of LN and ESRD over time, and mortality associated with renal disease.

Results At entry into the cohort, age of SLE patients [mean (SD)] was $27.3(9,1)$ years and 90\% were female. One-hundred thirty-one $(59 \%)$ patients developed lupus nephritis during 9.95 years of follow-up; incidence-rate 59/1000 py, most events (78\%) were developed within the first year of diagnosis. Patients with lupus nephritis had lower baseline BMI, less frequency of arthritis, and higher hypertension. There were no differences on age at lupus diagnosis, gender and baseline comorbidities between lupus patients with and without renal involvement. Among patients with renal biopsy, 80\% had ISN/ RPS Class IV and V alone or in combination. Twenty-eight (21\%) developed ESRD, five of them (18\%) have been received renal transplantation. Severe renal disease was strongly associated with poor outcomes in this cohort.

Conclusion LN in Hispanic SLE patients represents an early and severe manifestation with higher incidence. It imposes poorer prognosis during first years of disease duration.

\section{PS3:51 MULTIMORBIDITY BURDEN IN SLE: PRELIMINARY DATA FROM THE COMMUNITY-BASED LUPUS REGISTRY OF CRETE}

1,2I Gergianaki, ${ }^{1} \mathrm{C}$ Adamichou, ${ }^{1} \mathrm{G}$ Spyrou, 'A Kountouri, ${ }^{1,2}$ P Sidiropoulos, 2,3,4 D Boumpas, ${ }^{1,2} \mathrm{G}$ Bertsias. ${ }^{1}$ Rheumatology, Clinical Immunology and Allergy, University of Crete School of Medicine, Iraklio, Greece; ${ }^{2}$ Institute of Molecular Biology-Biotechnology, FORTH, Iraklio, Greece; ${ }^{3} 4$ th Department of Medicine, Attikon University Hospital, University of Athens Medical School, Athens, Greece; ${ }^{4}$ Biomedical Research Foundation of the Academy of Athens, Greece

10.1136/lupus-2018-abstract.99 